\title{
Subordination properties for a general class of integral operators involving meromorphically multivalent functions
}

Nak Eun $\mathrm{Cho}^{1 *}$ and Rekha Srivastava ${ }^{2}$

"Correspondence:

necho@pknu.ac.kr

'Department of Applied

Mathematics, Pukyong National

University, Busan, 608-737, Korea

Full list of author information is

available at the end of the article

\begin{abstract}
The purpose of the present paper is to investigate some subordination- and superordination-preserving properties of certain nonlinear integral operators defined on the space of meromorphically $p$-valent functions in the punctured open unit disk. The sandwich-type theorems associated with these integral operators are established. Relevant connections of the various results presented here with those involving relatively simpler nonlinear integral operators are also indicated.

MSC: Primary 30C45; secondary 30C80

Keywords: differential subordination; differential superordination; meromorphic functions; integral operators; convex functions; close-to-convex functions; subordination (or Löwner) chain
\end{abstract}

\section{Introduction, definitions and preliminaries}

Let $\mathcal{H}=\mathcal{H}(\mathbb{U})$ denote the class of analytic functions in the open unit disk

$$
\mathbb{U}=\{z: z \in \mathbb{C} \text { and }|z|<1\} .
$$

For $a \in \mathbb{C}$ and $n \in \mathbb{N}=\{1,2,3, \ldots\}$, let

$$
\mathcal{H}[a, n]=\left\{f \in \mathcal{H}: f(z)=a+a_{n} z^{n}+a_{n+1} z^{n+1}+\cdots\right\} .
$$

Let $f$ and $F$ be members of $\mathcal{H}$. The function $f$ is said to be subordinate to $F$, or $F$ is said to be superordinate to $f$, if there exists a function $w$ analytic in $\mathbb{U}$, with

$$
w(0)=0 \quad \text { and } \quad|w(z)|<1 \quad(z \in \mathbb{U})
$$

such that

$$
f(z)=F(w(z)) \quad(z \in \mathbb{U})
$$

In such a case, we write

$$
f \prec F \quad \text { or } \quad f(z) \prec F(z) \quad(z \in \mathbb{U}) \text {. }
$$

(0) 2013 Cho and Srivastava; licensee Springer. This is an Open Access article distributed under the terms of the Creative Commons Attribution License (http://creativecommons.org/licenses/by/2.0), which permits unrestricted use, distribution, and reproduction in any medium, provided the original work is properly cited. 
Furthermore, if the function $F$ is univalent in $\mathbb{U}$, then we have $(c f .[1,2]$ and $[3])$

$$
f \prec F \quad \Longleftrightarrow \quad f(0)=F(0) \text { and } f(\mathbb{U}) \subset F(\mathbb{U}) \text {. }
$$

Definition 1 (Miller and Mocanu [1]) Let

$$
\phi: \mathbb{C}^{2} \rightarrow \mathbb{C},
$$

and let $h$ be univalent in $\mathbb{U}$. If $\mathfrak{p}$ is analytic in $\mathbb{U}$ and satisfies the differential subordination

$$
\phi\left(\mathfrak{p}(z), z \mathfrak{p}^{\prime}(z)\right) \prec h(z) \quad(z \in \mathbb{U}),
$$

then $\mathfrak{p}$ is called a solution of the differential subordination. The univalent function $q$ is called a dominant of the solutions of the differential subordination or, more simply, a dominant if

$$
\mathfrak{p} \prec q \quad(z \in \mathbb{U})
$$

for all $\mathfrak{p}$ satisfying (1.1). A dominant $\tilde{q}$ that satisfies the following condition:

$$
\tilde{q} \prec q \quad(z \in \mathbb{U})
$$

for all dominants $q$ of (1.1) is said to be the best dominant.

Definition 2 (Miller and Mocanu [4]) Let

$$
\varphi: \mathbb{C}^{2} \rightarrow \mathbb{C}
$$

and let $h$ be analytic in $\mathbb{U}$. If $p$ and $\varphi\left(\mathfrak{p}(z), z \mathfrak{p}^{\prime}(z)\right)$ are univalent in $\mathbb{U}$ and satisfy the differential superordination

$$
h(z) \prec \varphi\left(\mathfrak{p}(z), z \mathfrak{p}^{\prime}(z)\right) \quad(z \in \mathbb{U}),
$$

then $\mathfrak{p}$ is called a solution of the differential superordination. An analytic function $q$ is called a subordinant of the solutions of the differential superordination or, more simply, a subordinant if

$$
q \prec \mathfrak{p} \quad(z \in \mathbb{U})
$$

for all $\mathfrak{p}$ satisfying (1.2). A univalent subordinant $\tilde{q}$ that satisfies the following condition:

$$
q \prec \tilde{q} \quad(z \in \mathbb{U})
$$

for all subordinants $q$ of (1.2) is said to be the best subordinant. 
Definition 3 (Miller and Mocanu [4]) We denote by $\mathcal{Q}$ the class of functions $f$ that are analytic and injective on $\overline{\mathbb{U}} \backslash E(f)$, where

$$
E(f)=\left\{\zeta: \zeta \in \partial \mathbb{U} \text { and } \lim _{z \rightarrow \zeta} f(z)=\infty\right\}
$$

and are such that

$$
f^{\prime}(\zeta) \neq 0 \quad(\zeta \in \partial \mathbb{U} \backslash E(f))
$$

We also denote the class $\mathcal{D}$ by

$$
\mathcal{D}:=\{\varphi \in \mathcal{H}[1,1]: \varphi(0)=1 \text { and } \varphi(z) \neq 0(z \in \mathbb{U})\}
$$

Let $\Sigma_{p}$ denote the class of functions of the form

$$
f(z)=\frac{1}{z^{p}}+\frac{a_{0}}{z^{p-1}}+\frac{a_{1}}{z^{p-2}}+\cdots+a_{n+p-1} z^{n}+\cdots \quad(p \in \mathbb{N})
$$

which are analytic in the punctured open unit disk $\mathbb{D}=\mathbb{U} \backslash\{0\}$. Let $\Sigma^{*}$ and $\Sigma_{k}$ be the subclasses of $\Sigma_{1}$ consisting of all functions which are, respectively, meromorphically starlike and meromorphically convex in $\mathbb{D}$ (see, for details, $[1,5]$ ).

For a function $f \in \Sigma_{p}$, we introduce the following general integral operator $I_{\alpha, \beta, \gamma, \delta}^{\phi, \varphi}$ defined by

$$
\begin{aligned}
& I_{\alpha, \beta, \gamma, \delta}^{\phi, \varphi}(f)(z):=\left(\frac{\gamma-p \beta}{z^{\gamma} \phi(z)} \int_{0}^{z} t^{\delta-1} f^{\alpha}(t) \varphi(t) d t\right)^{1 / \beta} \\
& \left(f \in \Sigma_{p} ; \alpha, \beta, \gamma, \delta \in \mathbb{C} ; \beta \in \mathbb{C} \backslash\{0\} ; \delta-p \alpha=\gamma-p \beta ; \Re\{\gamma-p \beta\}>0 ; \phi, \varphi \in \mathcal{D}\right) .
\end{aligned}
$$

Several members of the family of integral operators $I_{\alpha, \beta, \gamma, \delta}^{\phi, \varphi}(f)$ defined by (1) have been extensively studied by many authors (see, for example, [6-10]; see also [11] and [12]) with suitable restrictions on the parameters $\alpha, \beta, \gamma$ and $\delta$, and for $f$ belonging to some favored subclasses of meromorphic functions. In particular, Bajpai [6] showed that the integral operator $I_{1,2,1,1}^{1,1}(f)$ belongs to the classes $\Sigma^{*}$ and $\Sigma_{k}$, whenever $f$ belongs to the classes $\Sigma^{*}$ and $\Sigma_{k}$, respectively.

Making use of the principle of subordination between analytic functions, Miller et al. [13] and, more recently, Owa and Srivastava [14] obtained some interesting subordinationpreserving properties for certain integral operators. Moreover, Miller and Mocanu [4] considered differential superordinations as the dual concept of differential subordinations (see also [15]). It should be remarked that in recent years several authors obtained many interesting results involving various integral operators associated with differential subordination and superordination (for example, see [5,16-18]). In the present paper, we obtain the subordination- and superordination-preserving properties of the general integral operator $I_{\alpha, \beta, \gamma, \delta}^{\phi, \varphi}$ defined by (1) with the sandwich-type theorem.

The following lemmas will be required in our present investigation.

Lemma 1 (Miller and Mocanu [19]) Suppose that the function

$$
H: \mathbb{C}^{2} \rightarrow \mathbb{C}
$$


satisfies the following condition:

$$
\mathfrak{R}\{H(i s, t)\} \leqq 0
$$

for all real s and for all $t$ with

$$
t \leqq-\frac{n\left(1+s^{2}\right)}{2} \quad(n \in \mathbb{N}) .
$$

If the function

$$
\mathfrak{p}(z)=1+p_{n} z^{n}+\cdots
$$

is analytic in $\mathbb{U}$ and

$$
\mathfrak{R}\left\{H\left(\mathfrak{p}(z), z \mathfrak{p}^{\prime}(z)\right)\right\}>0 \quad(z \in \mathbb{U}),
$$

then

$$
\mathfrak{R}\{\mathfrak{p}(z)\}>0 \quad(z \in \mathbb{U}) .
$$

Lemma 2 (Miller and Mocanu [20]) Let

$$
\beta, \gamma \in \mathbb{C} \quad(\beta \neq 0) \quad \text { and } \quad h \in \mathcal{H}(\mathbb{U}) \quad(h(0)=c) .
$$

If

$$
\mathfrak{R}\{\beta h(z)+\gamma\}>0 \quad(z \in \mathbb{U}),
$$

then the solution of the differential equation

$$
q(z)+\frac{z q^{\prime}(z)}{\beta q(z)+\gamma}=h(z) \quad(z \in \mathbb{U} ; q(0)=c)
$$

is analytic in $\mathbb{U}$ and satisfies the following inequality:

$$
\Re\{\beta q(z)+\gamma\}>0 \quad(z \in \mathbb{U}) .
$$

Lemma 3 (Miller and Mocanu [1]) Let

$$
\mathfrak{p} \in \mathcal{Q} \quad(\mathfrak{p}(0)=a),
$$

and let

$$
q(z)=a+a_{n} z^{n}+\cdots
$$

be analytic in $\mathbb{U}$ with

$$
q(z) \not \equiv a \text { and } n \in \mathbb{N} \text {. }
$$


If the function $q$ is not subordinate to $\mathfrak{p}$, then there exist points

$$
z_{0}=r_{0} e^{i \theta} \in \mathbb{U} \text { and } \zeta_{0} \in \partial \mathbb{U} \backslash E(f),
$$

for which

$$
q\left(\mathbb{U}_{r_{0}}\right) \subset \mathfrak{p}(\mathbb{U}), \quad q\left(z_{0}\right)=\mathfrak{p}\left(\zeta_{0}\right) \quad \text { and } \quad z_{0} q^{\prime}\left(z_{0}\right)=m \zeta_{0} \mathfrak{p}^{\prime}\left(\zeta_{0}\right) \quad(m \geqq n)
$$

Let

$$
N:=N(c)=\frac{|c| \sqrt{1+2 \mathfrak{R}(c)}+\mathfrak{I}(c)}{\mathfrak{R}(c)} \quad(c \in \mathbb{C} ; \mathfrak{R}(c)>0) .
$$

If $R$ is the univalent function defined in $\mathbb{U}$ by

$$
R(z)=\frac{2 N z}{1-z^{2}} \quad(z \in \mathbb{U})
$$

then the open door function (see [1]) is defined by

$$
R_{c}(z):=R\left(\frac{z+b}{1+\bar{b} z}\right) \quad\left(b=R^{-1}(c) ; z \in \mathbb{U}\right) .
$$

Remark 1 The function $R_{c}$ defined by (1.4) is univalent in $\mathbb{U}$, where $R_{c}(0)=c$, and $R_{c}(\mathbb{U})=$ $R(\mathbb{U})$ is the complex plane with slits along the half-lines given by

$$
\mathfrak{R}(w)=0 \quad \text { and } \quad|\Im(w)| \geqq N .
$$

Lemma 4 (Totoi [21]) Let $\alpha, \beta, \gamma, \delta \in \mathbb{C}$ with

$$
\beta \neq 0, \quad \delta-p \alpha=\gamma-p \beta, \quad \Re\{\gamma-p \beta\}>0 \quad \text { and } \quad \phi, \varphi \in \mathcal{D} .
$$

Iff $\in \Sigma_{\alpha, \beta, \gamma, \delta}^{\phi, \varphi}$, where

$$
\Sigma_{\alpha, \beta, \gamma, \delta}^{\phi, \varphi}:=\left\{f \in \Sigma_{p}: \alpha \frac{z f^{\prime}(z)}{f(z)}+\frac{z \varphi^{\prime}(z)}{\varphi(z)}+\delta \prec R_{\delta-p \alpha}(z)\right\}
$$

and $R_{\delta-p \alpha}(z)$ is defined by (1.4) with $c=\delta-p \alpha$, then

$$
I_{\alpha, \beta, \gamma, \delta}^{\phi, \varphi}(f)(z) \in \Sigma_{p}, \quad z^{p} I_{\alpha, \beta, \gamma, \delta}^{\phi, \varphi}(f)(z) \neq 0 \quad(z \in \mathbb{U})
$$

and

$$
\mathfrak{R}\left\{\beta \frac{z\left(I_{\alpha, \beta, \gamma, \delta}^{\phi, \varphi}(f)(z)\right)^{\prime}}{I_{\alpha, \beta, \gamma, \delta}^{\phi, \varphi}(f)(z)}+\frac{z \phi^{\prime}(z)}{\phi(z)}+\gamma\right\}>0 \quad(z \in \mathbb{U}),
$$

where $I_{\alpha, \beta, \gamma, \delta}^{\phi, \varphi}$ is the integral operator defined by (1). 
A function $L(z, t)$ defined on $\mathbb{U} \times[0, \infty)$ is the subordination chain (or Löwner chain) if $L(\cdot, t)$ is analytic and univalent in $\mathbb{U}$ for all $t \in[0, \infty), L(z, \cdot)$ is continuously differentiable on $[0, \infty)$ for all $z \in \mathbb{U}$ and

$$
L(z, s) \prec L(z, t) \quad(z \in \mathbb{U} ; 0 \leqq s<t) .
$$

Lemma 5 (Miller and Mocanu [4]) Let

$$
q \in \mathcal{H}[a, 1] \text { and } \mu: \mathbb{C}^{2} \rightarrow \mathbb{C}
$$

Also set

$$
\mu\left(q(z), z q^{\prime}(z)\right)=: h(z) \quad(z \in \mathbb{U}) .
$$

If

$$
L(z, t)=\mu\left(q(z), t z q^{\prime}(z)\right)
$$

is a subordination chain and

$$
\mathfrak{p} \in \mathcal{H}[a, 1] \cap \mathcal{Q},
$$

then the following subordination condition:

$$
h(z) \prec \mu\left(\mathfrak{p}(z), z \mathfrak{p}^{\prime}(z)\right) \quad(z \in \mathbb{U})
$$

implies that

$$
q(z) \prec \mathfrak{p}(z) \quad(z \in \mathbb{U}) .
$$

Furthermore, if

$$
\mu\left(q(z), z q^{\prime}(z)\right)=h(z)
$$

has a univalent solution $q \in \mathcal{Q}$, then $q$ is the best subordinant.

Lemma 6 (Pommerenke [22]) The function

$$
L(z, t)=a_{1}(t) z+\cdots
$$

with

$$
a_{1}(t) \neq 0 \quad \text { and } \quad \lim _{t \rightarrow \infty}\left|a_{1}(t)\right|=\infty
$$

Suppose that $L(\cdot, t)$ is analytic in $\mathbb{U}$ for all $t \geqq 0$ and that $L(z, \cdot)$ is continuously differentiable on $[0, \infty)$ for all $z \in \mathbb{U}$. If the function $L(z, t)$ satisfies the following inequalities:

$$
\mathfrak{R}\left(\frac{\frac{z \partial L(z, t)}{\partial z}}{\frac{\partial L(z, t)}{\partial t}}\right)>0 \quad(z \in \mathbb{U} ; 0 \leqq t<\infty)
$$


and

$$
|L(z, t)| \leqq K_{0}\left|a_{1}(t)\right| \quad\left(|z|<r_{0}<1 ; 0 \leqq t<\infty\right)
$$

for some positive constants $K_{0}$ and $r_{0}$, then $L(z, t)$ is a subordination chain.

\section{Main results and their corollaries and consequences}

We begin by proving a general subordination property involving the integral operator $I_{\alpha, \beta, \gamma, \delta}^{\phi, \varphi}$ defined by (1), which is contained in Theorem 1 below.

Theorem 1 Let $f, g \in \Sigma_{\alpha, \beta, \gamma, \delta}^{\phi, \varphi}$, where $\Sigma_{\alpha, \beta, \gamma, \delta}^{\phi, \varphi}$ is defined by (1.5). Suppose also that

$$
\Re\left\{1+\frac{z v_{g}^{\prime \prime}(z)}{v_{g}^{\prime}(z)}\right\}>-\delta \quad\left(z \in \mathbb{U} ; v_{g}(z):=z\left(z^{p} g(z)\right)^{\alpha} \varphi(z)\right),
$$

where

$$
\rho=\frac{1+|\gamma-p \beta-1|^{2}-\left|1-(\gamma-p \beta-1)^{2}\right|}{4 \Re\{\gamma-p \beta-1\}} \quad(\Re\{\gamma-p \beta-1\}>0) .
$$

Then the subordination relation

$$
v_{f}(z) \prec v_{g}(z) \quad(z \in \mathbb{U})
$$

implies that

$$
z\left(z^{p} I_{\alpha, \beta, \gamma, \delta}^{\phi, \varphi}(f)(z)\right)^{\beta} \phi(z) \prec z\left(z^{p} I_{\alpha, \beta, \gamma, \delta}^{\phi, \varphi}(g)(z)\right)^{\beta} \phi(z) \quad(z \in \mathbb{U}),
$$

where $I_{\alpha, \beta, \gamma, \delta}^{\phi, \varphi}$ is the integral operator defined by (1). Moreover, the function

$$
z\left(z^{p} I_{\alpha, \beta, \gamma, \delta}^{\phi, \varphi}(g)(z)\right)^{\beta} \phi(z)
$$

is the best dominant.

Proof Let us define the functions $F$ and $G$, respectively, by

$$
F(z):=z\left(z^{p} I_{\alpha, \beta, \gamma, \delta}^{\phi, \varphi}(f)(z)\right)^{\beta} \phi(z) \quad \text { and } \quad G(z):=z\left(z^{p} I_{\alpha, \beta, \gamma, \delta}^{\phi, \varphi}(g)(z)\right)^{\beta} \phi(z) \text {. }
$$

We first show that if the function $q$ is defined by

$$
q(z):=1+\frac{z G^{\prime \prime}(z)}{G^{\prime}(z)} \quad(z \in \mathbb{U})
$$

then

$$
\mathfrak{R}\{q(z)\}>0 \quad(z \in \mathbb{U}) .
$$


From the definition of (1), we obtain

$$
\left(z^{p} I_{\alpha, \beta, \gamma, \delta}^{\phi, \varphi}(g)(z)\right)^{\beta} \phi(z)\left[\beta \frac{z\left(I_{\alpha, \beta, \gamma, \delta}^{\phi, \varphi}(g)(z)\right)^{\prime}}{I_{\alpha, \beta, \gamma, \delta}^{\phi, \varphi}(g)(z)}+\frac{z \phi^{\prime}(z)}{\phi(z)}+\gamma\right]=(\gamma-p \beta)\left(z^{p} g(z)\right)^{\alpha} \varphi(z) .
$$

We also have

$$
\beta \frac{z\left(I_{\alpha, \beta, \gamma, \delta}^{\phi, \varphi}(g)(z)\right)^{\prime}}{I_{\alpha, \beta, \gamma, \delta}^{\phi, \varphi}(g)(z)}+\frac{z \phi^{\prime}(z)}{\phi(z)}=\frac{z G^{\prime}(z)}{G(z)}-p \beta-1
$$

It follows from (2.7) and (2.8) that

$$
(\gamma-p \beta) v_{g}(z)=(\gamma-p \beta-1) G(z)+z G^{\prime}(z)
$$

Now, by a simple calculation with (2.9), we obtain the following relationship:

$$
1+\frac{z v_{g}^{\prime \prime}(z)}{v_{g}^{\prime}(z)}=q(z)+\frac{z q^{\prime}(z)}{q(z)+\gamma-p \beta-1} \equiv h(z)
$$

Thus, from (2.1), we have

$$
\mathfrak{R}\{h(z)+\gamma-p \beta-1\}>0 \quad(z \in \mathbb{U})
$$

and by using Lemma 2, we conclude that the differential equation (2.10) has a solution $q \in \mathcal{H}(\mathbb{U})$ with

$$
q(0)=h(0)=1
$$

Put

$$
H(u, v)=u+\frac{v}{u+\gamma-p \beta-1}+\rho,
$$

where $\rho$ is given by (2.2). From (2.1), (2.10) and (2.11), we obtain

$$
\mathfrak{R}\left\{H\left(q(z), z q^{\prime}(z)\right)\right\}>0 \quad(z \in \mathbb{U}) .
$$

We now proceed to show that

$$
\mathfrak{R}\{H(i s, t)\} \leqq 0 \quad\left(s \in \mathbb{R} ; t \leqq-\frac{\left(1+s^{2}\right)}{2}\right)
$$

Indeed, from (2.11), we have

$$
\begin{aligned}
\Re\{H(i s, t)\} & =\Re\left\{i s+\frac{t}{i s+\gamma-p \beta-1}+\rho\right\} \\
& =\frac{t \Re\{\gamma-p \beta-1\}}{|\gamma-p \beta-1+i s|^{2}}+\rho \\
& \leq-\frac{E_{\rho}(s)}{2|\gamma-p \beta-1+i s|^{2}},
\end{aligned}
$$


where

$$
\begin{aligned}
E_{\rho}(s):= & (\mathfrak{R}\{\gamma-p \beta-1\}-2 \rho) s^{2}-4 \rho(\Im\{\gamma-p \beta-1\}) s \\
& -2 \rho|\gamma-p \beta-1|^{2}+\mathfrak{R}\{\gamma-p \beta-1\} .
\end{aligned}
$$

For $\rho$ given by (2.2), we note that the coefficient of $s^{2}$ in the quadratic expression $E_{\rho}(s)$ given by (2.14) is positive or equal to zero and also $E_{\rho}(s)$ is a perfect square. Hence from (2.13), we see that (2.12) holds true. Thus, by using Lemma 1 , we conclude that

$$
\mathfrak{R}\{q(z)\}>0 \quad(z \in \mathbb{U})
$$

That is, the function $G(z)$ defined by $(2.5)$ is convex in $\mathbb{U}$.

We next prove that the subordination condition (2.3) implies that

$$
F(z) \prec G(z) \quad(z \in \mathbb{U})
$$

for the functions $F$ and $G$ defined by (2.5). Without loss of generality, we can assume that $G$ is analytic and univalent on $\overline{\mathbb{U}}$ and that

$$
F^{\prime}(\zeta) \neq 0 \quad(|\zeta|=1)
$$

We now consider the function $L(z, t)$ defined by

$$
L(z, t):=\frac{\gamma-p \beta-1}{\gamma-p \beta} G(z)+\frac{1+t}{\gamma-p \beta} z G^{\prime}(z) \quad(z \in \mathbb{U} ; 0 \leq t<\infty) .
$$

We note that

$$
\left.\frac{\partial L(z, t)}{\partial z}\right|_{z=z_{0}}=G^{\prime}(0)\left(1+\frac{t}{\gamma-p \beta}\right) \neq 0 \quad(z \in \mathbb{U} ; 0 \leq t<\infty)
$$

and

$$
\mathfrak{R}\left\{\frac{z \partial L(z, t) / \partial z}{\partial L(z, t) / \partial t}\right\}=\mathfrak{R}\left\{\gamma-p \beta-1+(1+t)\left(1+\frac{z G^{\prime \prime}(z)}{G^{\prime}(z)}\right)\right\}>0 \quad(z \in \mathbb{U}) .
$$

Furthermore, since $G$ is convex, by using the well-known growth and distortion sharp inequalities for convex functions (see [23]), we can prove that the second condition of Lemma 6 is satisfied. Therefore, by virtue of Lemma $6, L(z, t)$ is a subordination chain. We observe from the definition of a subordination chain that

$$
v_{g}(z)=\frac{\gamma-p \beta-1}{\gamma-p \beta} G(z)+\frac{1}{\gamma-p \beta} z G^{\prime}(z)=L(z, 0)
$$

and

$$
L(z, 0) \prec L(z, t) \quad(z \in \mathbb{U} ; 0 \leq t<\infty) .
$$


This implies that

$$
L(\zeta, t) \notin L(\mathbb{U}, 0)=v_{g}(\mathbb{U}) \quad(\zeta \in \partial \mathbb{U} ; 0 \leq t<\infty) .
$$

We now suppose that $F$ is not subordinate to $G$. Then, in view of Lemma 3, there exist points $z_{0} \in \mathbb{U}$ and $\zeta_{0} \in \partial \mathbb{U}$ such that

$$
F\left(z_{0}\right)=G\left(\zeta_{0}\right) \quad \text { and } \quad z_{0} F\left(z_{0}\right)=(1+t) \zeta_{0} G^{\prime}\left(\zeta_{0}\right) \quad(0 \leq t<\infty)
$$

Hence we have

$$
\begin{aligned}
L\left(\zeta_{0}, t\right) & =\frac{\gamma-p \beta-1}{\gamma-p \beta} G\left(\zeta_{0}\right)+\frac{1+t}{\gamma-p \beta} \zeta_{0} G^{\prime}\left(\zeta_{0}\right) \\
& =\frac{\gamma-p \beta-1}{\gamma-p \beta} F\left(\zeta_{0}\right)+\frac{1}{\gamma-p \beta} z_{0} F^{\prime}\left(z_{0}\right) \\
& =v_{f}\left(z_{0}\right) \in v_{g}(\mathbb{U})
\end{aligned}
$$

by virtue of the subordination condition (2.3). This contradicts the above observation that

$$
L\left(\zeta_{0}, t\right) \notin v_{g}(\mathbb{U})
$$

Therefore, the subordination condition (2.3) must imply the subordination given by (2.15). Considering $F=G$, we see that the function $G$ is the best dominant. This evidently completes the proof of Theorem 1 .

We next prove a solution to a dual problem of Theorem 1 in the sense that the subordinations are replaced by superordinations.

Theorem 2 Let $f, g \in \Sigma_{\alpha, \beta, \gamma, \delta}^{\phi, \varphi}$, where $\Sigma_{\alpha, \beta, \gamma, \delta}^{\phi, \varphi}$ is defined by (1.5). Suppose also that

$$
\Re\left\{1+\frac{z v_{g}^{\prime \prime}(z)}{v_{g}^{\prime}(z)}\right\}>-\rho \quad\left(z \in \mathbb{U} ; v_{g}(z):=z\left(z^{p} g(z)\right)^{\alpha} \varphi(z)\right) \text {, }
$$

where $\rho$ is given by (2.2) and $v_{f}$ is univalent in $\mathbb{U}$, and

$$
z\left(z^{p} I_{\alpha, \beta, \gamma, \delta}^{\phi, \varphi}(f)(z)\right)^{\beta} \phi(z) \in \mathcal{Q},
$$

where $I_{\alpha, \beta, \gamma, \delta}^{\phi, \varphi}$ is the integral operator defined by (1). Then the superordination relation

$$
v_{g}(z) \prec v_{f}(z) \quad(z \in \mathbb{U})
$$

implies that

$$
z\left(z^{p} I_{\alpha, \beta, \gamma, \delta}^{\phi, \varphi}(g)(z)\right)^{\beta} \phi(z) \prec z\left(z^{p} I_{\alpha, \beta, \gamma, \delta}^{\phi, \varphi}(f)(z)\right)^{\beta} \phi(z) \quad(z \in \mathbb{U}) .
$$

Moreover, the function

$$
z\left(z^{p} I_{\alpha, \beta, \gamma, \delta}^{\phi, \varphi}(g)(z)\right)^{\beta} \phi(z)
$$

is the best subordinant. 
Proof Let the functions $F$ and $G$ be given by (2.5). We first note from (2.7) and (2.8) that

$$
\begin{aligned}
v_{g}(z) & =\frac{\gamma-p \beta-1}{\gamma-p \beta} G(z)+\frac{1}{\gamma-p \beta} z G^{\prime}(z) \\
& =: \mu\left(G^{\prime}(z), z G^{\prime}(z)\right) .
\end{aligned}
$$

After a simple calculation, equation (2.17) yields the following relationship:

$$
1+\frac{z v_{g}^{\prime \prime}(z)}{v_{g}^{\prime}(z)}=q(z)+\frac{z q^{\prime}(z)}{q(z)+\gamma-p \beta-1}
$$

where the function $q$ is given in (2.6). Then, by using the same method as in the proof of Theorem 1, we can prove that

$$
\mathfrak{R}\{q(z)\}>0 \quad(z \in \mathbb{U}),
$$

that is, $G$ defined by (2.5) is convex (univalent) in $\mathbb{U}$.

We next prove that the superordination condition (2.16) implies that

$$
G(z) \prec F(z) \quad(z \in \mathbb{U}) .
$$

For this purpose, we consider the function $L(z, t)$ defined by

$$
L(z, t):=\frac{\gamma-p \beta-1}{\gamma-p \beta} G(z)+\frac{t}{\gamma-p \beta} z G^{\prime}(z) \quad(z \in \mathbb{U} ; 0 \leq t<\infty) .
$$

Since $G$ is convex and $\mathfrak{R}\{\gamma-p \beta-1\}>0$, we can prove easily that $L(z, t)$ is a subordination chain as in the proof of Theorem 1 . Therefore, according to Lemma 5, we conclude that the superordination condition (2.16) must imply the superordination given by (2.18). Furthermore, since the differential equation (2.17) has the univalent solution $G$, it is the best subordinant of the given differential superordination. Hence we complete the proof of Theorem 2.

If we combine Theorem 1 and Theorem 2, then we obtain the following sandwich-type theorem.

Theorem 3 Letf,$g_{k} \in \Sigma_{\alpha, \beta, \gamma, \delta}^{\phi, \varphi}(k=1,2)$, where $\Sigma_{\alpha, \beta, \gamma, \delta}^{\phi, \varphi}$ is defined by (1.5). Suppose also that

$$
\mathfrak{R}\left\{1+\frac{z v_{g_{k}}^{\prime \prime}(z)}{v_{g_{k}}^{\prime}(z)}\right\}>-\rho \quad\left(z \in \mathbb{U} ; v_{g_{k}}(z):=z\left(z^{p} g_{k}(z)\right)^{\alpha} \varphi(z) ; k=1,2\right),
$$

where $\rho$ is given by (2.2) and the function $v_{f}$ is univalent in $\mathbb{U}$, and

$$
z\left(z^{p} I_{\alpha, \beta, \gamma, \delta}^{\phi, \varphi}(f)(z)\right)^{\beta} \phi(z) \in \mathcal{Q},
$$

where $I_{\alpha, \beta, \gamma, \delta}^{\phi, \varphi}$ is the integral operator defined by (1). Then the subordination relation

$$
v_{g_{1}}(z) \prec v_{f}(z) \prec v_{g_{2}}(z) \quad(z \in \mathbb{U})
$$


implies that

$$
\begin{aligned}
z\left(z^{p} I_{\alpha, \beta, \gamma, \delta}^{\phi, \varphi}\left(g_{1}\right)(z)\right)^{\beta} \phi(z) & \prec z\left(z^{p} I_{\alpha, \beta, \gamma, \delta}^{\phi, \varphi}(f)(z)\right)^{\beta} \phi(z) \\
& \prec z\left(z^{p} I_{\alpha, \beta, \gamma, \delta}^{\phi, \varphi}\left(g_{2}\right)(z)\right)^{\beta} \phi(z) \quad(z \in \mathbb{U}) .
\end{aligned}
$$

Moreover, the functions

$$
z\left(z^{p} I_{\alpha, \beta, \gamma, \delta}^{\phi, \varphi}\left(g_{1}\right)(z)\right)^{\beta} \phi(z) \quad \text { and } \quad z\left(z^{p} I_{\alpha, \beta, \gamma, \delta}^{\phi, \varphi}\left(g_{2}\right)(z)\right)^{\beta} \phi(z)
$$

are the best subordinant and the best dominant, respectively.

The assumption of Theorem 3, that is, the functions

$$
z\left(z^{p} f(z)\right)^{\alpha} \varphi(z) \quad \text { and } \quad z\left(z^{p} I_{\alpha, \beta, \gamma, \delta}^{\phi, \varphi}(f)(z)\right)^{\beta} \phi(z)
$$

need to be univalent in $\mathbb{U}$, will be replaced by another set of conditions in the following result.

Corollary 1 Letf,$g_{k} \in \Sigma_{\alpha, \beta, \gamma, \delta}^{\phi, \varphi}(k=1,2)$, where $\Sigma_{\alpha, \beta, \gamma, \delta}^{\phi, \varphi}$ is defined by (1.5). Suppose also that the condition (2.19) is satisfied and

$$
\Re\left\{1+\frac{z v_{f}^{\prime \prime}(z)}{v_{f}^{\prime}(z)}\right\}>-\rho \quad\left(z \in \mathbb{U} ; v_{f}(z):=z\left(z^{p} f(z)\right)^{\alpha} \varphi(z) ; z f(z) \in \mathcal{Q}\right),
$$

where $\rho$ is given by (2.2). Then the subordination relation

$$
v_{g_{1}}(z) \prec v_{f}(z) \prec v_{g_{2}}(z) \quad(z \in \mathbb{U})
$$

implies that

$$
\begin{aligned}
z\left(z^{p} I_{\alpha, \beta, \gamma, \delta}^{\phi, \varphi}\left(g_{1}\right)(z)\right)^{\beta} \phi(z) & \prec z\left(z^{p} I_{\alpha, \beta, \gamma, \delta}^{\phi, \varphi}(f)(z)\right)^{\beta} \phi(z) \\
& \prec z\left(z^{p} I_{\alpha, \beta, \gamma, \delta}^{\phi, \varphi}\left(g_{2}\right)(z)\right)^{\beta} \phi(z) \quad(z \in \mathbb{U}),
\end{aligned}
$$

where $I_{\alpha, \beta, \gamma, \delta}^{\phi, \varphi}$ is the integral operator defined by (1). Moreover, the functions

$$
z\left(z^{p} I_{\alpha, \beta, \gamma, \delta}^{\phi, \varphi}\left(g_{1}\right)(z)\right)^{\beta} \phi(z) \quad \text { and } \quad z\left(z^{p} I_{\alpha, \beta, \gamma, \delta}^{\phi, \varphi}\left(g_{2}\right)(z)\right)^{\beta} \phi(z)
$$

are the best subordinant and the best dominant, respectively.

Proof In order to prove Corollary 1, we have to show that the condition (2.20) implies the univalence of $v_{f}(z)$ and

$$
F(z):=z\left(z^{p} I_{\alpha, \beta, \gamma, \delta}^{\phi, \varphi}(f)(z)\right)^{\beta} \phi(z)
$$

By noting that $0<\rho \leqq 1 / 2$ from (2.2), we obtain from the condition (2.20) that $\psi$ is a close-to-convex function in $\mathbb{U}$ (see [24]), and hence $v_{f}$ is univalent in $\mathbb{U}$. Furthermore, 
by using the same techniques as in the proof of Theorem 3, we can prove the convexity (univalence) of $F$ and so the details may be omitted. Therefore, by applying Theorem 3, we obtain Corollary 1.

By setting $\gamma-p \beta=3$ in Theorem 3, we have the following result.

Corollary 2 Let $f, g_{k} \in \Sigma_{\alpha, \beta, p \beta+3, \delta}^{\phi, \varphi}(k=1,2)$, where $\Sigma_{\alpha, \beta, p \beta+3, \delta}^{\phi, \varphi}$ is defined by (1.5) with $\gamma=$ $p \beta+3$. Suppose also that

$$
\Re\left\{1+\frac{z v_{g_{k}}^{\prime \prime}(z)}{v_{g_{k}}^{\prime}(z)}\right\}>-\frac{1}{4} \quad\left(z \in \mathbb{U} ; v(z):=z\left(z^{p} g_{k}(z)\right)^{\alpha} \varphi(z) ; k=1,2\right),
$$

and the function $v_{f}$ is univalent in $\mathbb{U}$, and

$$
z\left(z^{p} I_{\alpha, \beta, p \beta+3, \delta}^{\phi, \varphi}(f)(z)\right)^{\beta} \phi(z) \in \mathcal{Q},
$$

where $I_{\alpha, \beta, p \beta+3, \delta}^{\phi, \varphi}$ is the integral operator defined by (1) with $\gamma=p \beta+3$. Then the subordination relation

$$
v_{g_{1}} \prec v_{f} \prec v_{g_{2}} \quad(z \in \mathbb{U})
$$

implies that

$$
\begin{aligned}
z\left(z^{p} I_{\alpha, \beta, p \beta+3, \delta}\left(g_{1}\right)(z)\right)^{\beta} \phi(z) & \prec z\left(z^{p} I_{\alpha, \beta, p \beta+3, \delta}(f)(z)\right)^{\beta} \phi(z) \\
& \prec z\left(z^{p} I_{\alpha, \beta, p \beta+3, \delta}\left(g_{2}\right)(z)\right)^{\beta} \phi(z) \quad(z \in \mathbb{U}) .
\end{aligned}
$$

Moreover, the functions

$$
z\left(z^{p} I_{\alpha, \beta, p \beta+3, \delta}\left(g_{1}\right)(z)\right)^{\beta} \phi(z) \quad \text { and } \quad z\left(z^{p} I_{\alpha, \beta, p \beta+3, \delta}\left(g_{2}\right)(z)\right)^{\beta} \phi(z)
$$

are the best subordinant and the best dominant, respectively.

If we take $\gamma-p \beta=2+i$ in Theorem 3, then we are easily led to the following result.

Corollary 3 Let $f, g_{k} \in \Sigma_{\alpha, \beta, p \beta+2+i, \delta}^{\phi, \varphi}(k=1,2)$, where $\Sigma_{\alpha, \beta, p \beta+2+i, \delta}^{\phi, \varphi}$ is defined by (1.5) with $\gamma=p \beta+2+i$. Suppose also that

$$
\begin{aligned}
& \mathfrak{R}\left\{1+\frac{z v_{g_{k}}^{\prime \prime}(z)}{v_{g_{k}}^{\prime}(z)}\right\}>-\frac{3-\sqrt{5}}{4} \\
& \left(z \in \mathbb{U} ; v_{g_{k}}(z):=z\left(z^{p} g_{k}(z)\right)^{\alpha} \varphi(z) ; k=1,2\right),
\end{aligned}
$$

and the function $v_{f}$ is univalent in $\mathbb{U}$, and

$$
z\left(z^{p} I_{\alpha, \beta, p \beta+2+i, \delta}^{\phi, \varphi}(f)(z)\right)^{\beta} \phi(z) \in \mathcal{Q},
$$


where $I_{\alpha, \beta, p \beta+2+i, \delta}^{\phi, \varphi}$ is the integral operator defined by (1) with $\gamma=p \beta+2+i$. Then the subordination relation

$$
v_{g_{1}} \prec v_{f} \prec v_{g_{2}} \quad(z \in \mathbb{U})
$$

implies that

$$
\begin{aligned}
z\left(z^{p} I_{\alpha, \beta, p \beta+2+i, \delta}^{\phi, \varphi}\left(g_{1}\right)(z)\right)^{\beta} \phi(z) & \prec z\left(z^{p} I_{\alpha, \beta, p \beta+2+i, \delta}^{\phi, \varphi}(f)(z)\right)^{\beta} \phi(z) \\
& \prec z\left(z^{p} I_{\alpha, \beta, p \beta+2+i, \delta}^{\phi, \varphi}\left(g_{2}\right)(z)\right)^{\beta} \phi(z) \quad(z \in \mathbb{U}) .
\end{aligned}
$$

Moreover, the functions

$$
z\left(z^{p} I_{\alpha, \beta, p \beta+2+i, \delta}^{\phi, \varphi}\left(g_{1}\right)(z)\right)^{\beta} \phi(z) \quad \text { and } \quad z\left(z^{p} I_{\alpha, \beta, p \beta+2+i, \delta}^{\phi, \varphi}\left(g_{2}\right)(z)\right)^{\beta} \phi(z)
$$

are the best subordinant and the best dominant, respectively.

\section{Competing interests}

The authors declare that they have no competing interests.

\section{Authors' contributions}

Both authors jointly worked on the results and they read and approved the final manuscript.

\section{Author details}

'Department of Applied Mathematics, Pukyong National University, Busan, 608-737, Korea. ${ }^{2}$ Department of Mathematics and Statistics, University of Victoria, Victoria, BC V8W 3R4, Canada.

\section{Acknowledgements}

Dedicated to Professor Hari M Srivastava.

This research was supported by the Basic Science Research Program through the National Research Foundation of the Republic of Korea (NRF) funded by the Ministry of Education, Science and Technology (No. 2012-0002619).

\section{Received: 14 January 2013 Accepted: 14 March 2013 Published: 5 April 2013}

\section{References}

1. Miller, SS, Mocanu, PT: Differential Subordinations, Theory and Applications. Dekker, New York (2000)

2. Srivastava, HM, Owa, S (eds.): Univalent Functions, Fractional Calculus, and Their Applications. Wiley, New York (1989)

3. Srivastava, HM, Owa, S (eds.): Current Topics in Analytic Function Theory. World Scientific, Singapore (1992)

4. Miller, SS, Mocanu, PT: Subordinants of differential superordinations. Complex Var. Theory Appl. 48, $815-826$ (2003)

5. Bulboacă, T: Differential subordination and superordination-preserving integral operators. Trans. Inst. Math. Nat. Acad. Sci. Ukraine 3, 19-28 (2004)

6. Bajpai, SK: A note on a class of meromorphic univalent functions. Rev. Roum. Math. Pures Appl. 22, $295-297$ (1977)

7. Bhoosnurmath, SS, Swamy, SR: Certain integrals for classes of univalent meromorphic functions. Ganit 44, 19-25 (1993)

8. Dernek, A: Certain classes of meromorphic functions. Ann. Univ. Mariae Curie-SkTlodowska, Sect. A 42, 1-8 (1988)

9. Dwivedi, SP, Bhargava, GP, Shukla, SK: On some classes of meromorphic univalent functions. Rev. Roum. Math. Pures Appl. 25, 209-215 (1980)

10. Goel, RM, Sohi, NS: On a class of meromorphic functions. Glas. Mat. 17(37), 19-28 (1981)

11. Sokół, J, Noor, KI, Srivastava, HM: A family of convolution operators for multivalent analytic functions. Eur. J. Pure Appl. Math. 5, 469-479 (2012)

12. Srivastava, HM, Yang, D-G, Xu, N-E: Subordinations for multivalent analytic functions associated with the Dziok-Srivastava operator. Integral Transforms Spec. Funct. 20, 581-606 (2009)

13. Miller, SS, Mocanu, PT, Reade, MO: Subordination-preserving integral operators. Trans. Am. Math. Soc. 283, 605-615 (1984)

14. Owa, S, Srivastava, HM: Some subordination theorems involving a certain family of integral operators. Integral Transforms Spec. Funct. 15, 445-454 (2004)

15. Bulboacă, T: A class of superordination-preserving integral operators. Indag. Math. 13, 301-311 (2002)

16. Bulboacă, T: Sandwich-type results for a class of convex integral operators. Acta Math. Sci. 32B, $989-1001$ (2012)

17. Cho, NE, Srivastava, HM: A class of nonlinear integral operators preserving subordination and superordination. Integral Transforms Spec. Funct. 18, 95-107 (2007)

18. Cho, NE, Bulboaca, T, Srivastava, HM: A general family of integral operators and associated subordination and superordination properties of some special analytic function classes. Appl. Math. Comput. 219, 2278-2288 (2012) 
19. Miller, SS, Mocanu, PT: Differential subordinations and univalent functions. Mich. Math. J. 28, 157-171 (1981)

20. Miller, SS, Mocanu, PT: Univalent solutions of Briot-Bouquet differential equations. J. Differ. Equ. 56, 297-309 (1985)

21. Totoi, A: On some classes of meromorphic functions defined by a multiplier transformation. Acta Univ. Apulensis, Mat-Inform. 25, 41-52 (2011)

22. Pommerenke, C: Univalent Functions. Vanderhoeck \& Ruprecht, Göttingen (1975)

23. Hallenbeck, DJ, MacGregor, TH: Linear Problems and Convexity Techniques in Geometric Function Theory. Pitman, London (1984)

24. Kaplan, W: Close-to-convex schlicht functions. Mich. Math. J. 2, 169-185 (1952)

doi:10.1186/1687-1847-2013-93

Cite this article as: Cho and Srivastava: Subordination properties for a general class of integral operators involving meromorphically multivalent functions. Advances in Difference Equations 2013 2013:93.

Submit your manuscript to a SpringerOpen ${ }^{\odot}$ journal and benefit from:

- Convenient online submission

- Rigorous peer review

- Immediate publication on acceptance

- Open access: articles freely available online

- High visibility within the field

- Retaining the copyright to your article 